\title{
Virtual Worlds for Facilitating Urban Development Dialogue
}

\author{
David Harris Smith \\ McMaster University \\ 1280 Main St W, Hamilton, ON \\ Canada \\ dhsmith@mcmaster.ca
}

\author{
Débora Silva de Jesus \\ McMaster University \\ 1280 Main St W, Hamilton, ON \\ Canada \\ deborajesus@gmail.com
}

\author{
Frauke Zeller \\ Ryerson University \\ 350 Victoria St, Toronto, ON \\ Canada \\ fzeller@ryerson.ca \\ Lauren Dwyer \\ Ryerson University \\ 350 Victoria St, Toronto, ON \\ Canada \\ laurenjdwyer@gmail.com
}

\author{
John Eyles \\ McMaster University \\ 1280 Main St W, Hamilton, ON, \\ Canada \\ eyles@mcmaster.ca
}

\author{
Emily Eyles \\ University of Bristol \\ Tyndall Ave, Bristol BS8 1TH \\ UK \\ ec.eyles@gmail.com
}

\section{INTRODUCTION}

As digital technologies have come to shape the city and the daily lives of its citizenry (Ridell \& Zeller 2013), it is timely and necessary that the means by which we envision the future city are enabled by these same technologies. Batty (1997) suggests "that computers will have to be used to understand cities which are built of computers. There will be no other way" (p. 159). Yet, planning activities are not easily communicated, nor readily understood by all stakeholders (Podevyn et al. 2009, Ylipulli \& Suopajärvi 2013). Furthermore, there is currently a gap in the research on how Web 2.0 technologies, and especially, virtual worlds, may be effectively utilised to achieve fuller civic participation in planning activities.

This paper documents multiple method interdisciplinary research to develop and test virtual world technologies for participatory urban planning and design. The researchers collaborated with the City of Hamilton (Canada) to design and develop prototype Opensimulator (opensimulator.org) virtual environments to be employed in public planning charrettes. The objective of the research was to test the efficacy of 3D avatar virtual worlds for communicating changes to urban environments. The results presented are derived from the use of virtual worlds used in two cases of urban development, including the simulation of a proposed Light Rail Transit (LRT) platform and the simulation of streetscape modifications.

\section{METHOD}

We applied user-centered interaction design methodology, incorporating focus groups and expert interviews, concept modeling, virtual system development, user studies and public charrette implementation. Stakeholder focus groups and expert interviews were used to gain insight into urban planning opinions and experiences from the perspective of residents, business owners, municipal officials, and professional planners. Interview and focus group participants were introduced to a preliminary version of the virtual planning environment to elicit comments and suggestions for features and applications. Findings from these methods were then used to establish design needs and model concepts for integration in the virtual planning environments for the two case studies.

\subsection{Complete Streets Design}

In the first case, the virtual world was used to communicate the proposed implementation of Complete Streets (completestreetsforcanada.ca), a planning concept incorporating inclusive street design for all types of users. The Opensimulator virtual environment was supplemented by Streetmix (streetmix.net), an open-source street 
design software. The combined platform allowed users to specify street features in Streetmix through drag and drop operations, which were updated in real-time in the virtual environment.

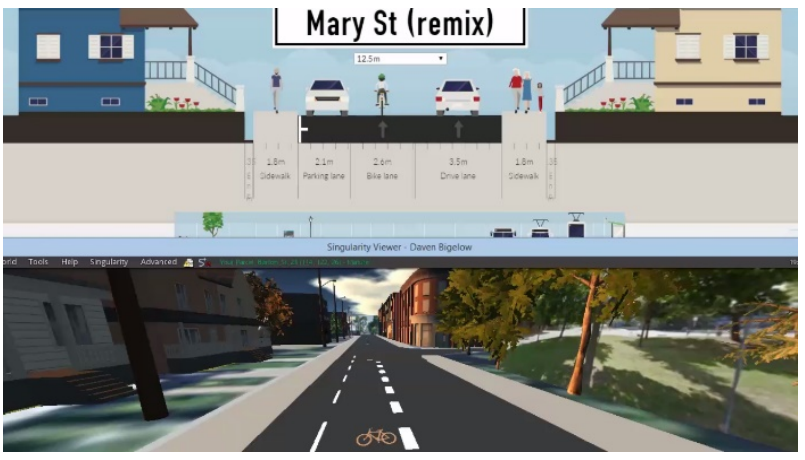

Figure 1: Complete Streets Design using Streetmix and Opensimulator. Street design choices selected in upper window are automatically updated in lower virtual environment window

A cost calculator was implemented in the virtual environment to tally the cost of street renovations selected by the user. The model was incorporated in two public planning charrettes (100 attendees each), where participants worked in small groups (6-10), alternately with the virtual planning environment and tabletop analog maps with moveable cutouts to simulate streetscape changes.

\subsection{LRT Design}

In the second case, the avatar virtual world was used to communicate street designs for access to a proposed Light Rail Transit system (LRT). The LRT model was subjected to a user study in which 10 participants were invited to perform a list of set tasks in the LRT simulation, providing feedback on the efficacy of the virtual world and avatar for visualising the proposed LRT system.

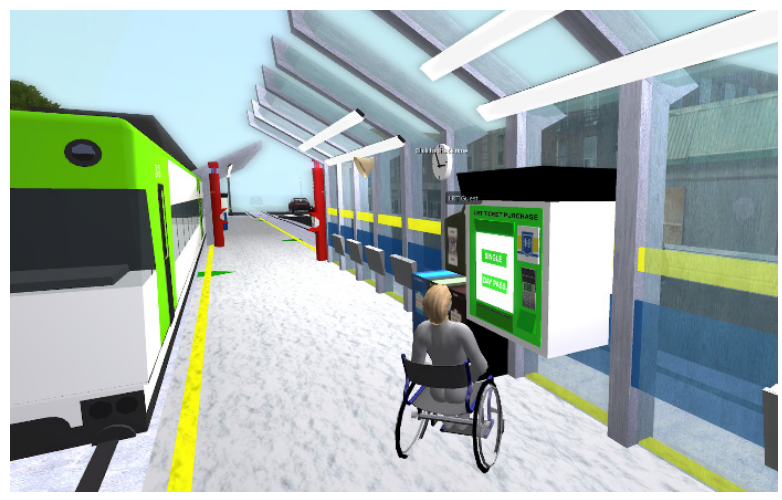

Figure 2: Avatar wheelchair user interacting with $L R T$ simulation.

The LRT simulation incorporated Universal Design (UD) accessibility features, allowing users to navigate the virtual environment using an avatar with or without a wheelchair. User study participants $(\mathrm{N}=10)$ were invited to perform a list of tasks in the scenario, such as moving from the sidewalk to the LRT platform, buying a ticket, and boarding the LRT.

A video game joystick controller was introduced as an alternate navigation and control interface in repeat trials. Audio and video screen capture was used to record user interactions and think aloud narration. Participant verbal feedback was complemented by a questionnaire used to evaluate overall experience, and specific evaluations related to navigation efficiency, information accessibility, and simulation effectiveness.

\section{RESULTS}

The expert interviews and focus groups indicated that while participatory planning has been readily adopted as a value by municipal authorities and planning professionals, it has not been successfully implemented. Problems cited by professionals include the increased costs and workload to facilitate public participation, planning knowledge deficits on the part of the lay participants.

Lay participants and business operators cited a lack of information from planning authorities and professionals and the offer of token, rather than meaningful, planning engagement. All participants responded positively to the virtual planning environment, requesting ongoing access to the environment and suggesting extended application to other planning sites and issues.

Results of the user studies and public charrettes, indicate that while interactive virtual world visualisation improves the communication of urban design to citizen stakeholders, the user interface design of the Opensimulator virtual world, specifically the default configuration of avatar navigation controls, camera point of view, and contextual menu options, presents significant challenges to non-expert users. This challenge was overcome in the public charrettes by having an experienced individual navigate and make changes to the virtual environment at the request of stakeholders.

Additionally, screen projection or large monitor viewing of the virtual planning environment, was essential for multiple participants engaged simultaneously in a planning task. Where only a desktop monitor or laptop display was available for visualising planning changes, sustained group attention to the planning task was difficult to maintain and participants preferred to work with the alternate tabletop analog maps and cutouts. 


\section{REFERENCES}

Batty, M. (1997). The computable city. International Planning Studies, 2(2), pp. 155-173.

Ridell, S. and Zeller, F. (2013). Mediated urbanism: Navigating an interdisciplinary terrain. The International Communication Gazette, 75(5-6), pp. 437-451.

Smith, D. H. (2013) Reflexive design of the recursive space of virtual worlds. International Communication Gazette, 75(5-6), pp. 452-469.
Podevyn, M., Horne, M., and Fisher, P. (2009) Virtual cities: Management and organisational issues. CUPUM 2009 11th International Conference on Computers in Urban Planning and Urban Management, Hong Kong, June 2009, pp. $1-13$.

Ylipulli J. and Suopajärvi T. (2013) Contesting ubicomp visions through ICT practices: Power negotiations in the meshwork of a technologized city. International Communication Gazette, 75(5-6), pp. 538-554. 